\title{
How has the Internet affected dentistry?
}

\author{
Perceptions of how the Internet has impacted on dentistry
}

\section{G. Chestnutt and K. Reynolds Br Dent J 2006; 200: 161-165}

\section{Objectives}

This study sought to identify how patient information on the Internet has influenced the delivery of oral care and the use practitioners themselves make of the Internet.

\section{Methodology}

Data were collected via a self completed 18 item postal questionnaire, sent to a random sample of 620 dentists on the GDC register in Wales. Results

In total 457 (74\%) of the questionnaires were returned. One half of all practitioners stated that patients had asked them about material of relevance to dentistry obtained from the Internet, although in the majority of cases this happened less frequently than once a month. The most common topics enquired after were cosmetic procedures, dental amalgam and implants. A minority of dentists, 47 (11\%) viewed the Internet as a threat to the dentist-patient relationship. However, 169 (39\%) agreed information gained from the Internet had led to patients demanding inappropriate care or more complex treatment (135/31\%). Having to take time to discuss Internet material was viewed as a burden by 93 practitioners. The potential of the Internet to widen inequalities in access to oral health information was agreed upon by one third of respondents. Concerns over the quality and reliability of Internet derived information, together with a lack of knowledge of appropriate sites, prevented dentists using the Internet as an oral health education resource. However, only 18.7\% claimed never to use the Internet for their own Continuing Professional Development. Access for CPD purposes was mainly from home. Of those working in general practice, 54 had their own practice website, and a further 103 said this was something they were considering. Email was used to communicate with patients by 42 dentists, mainly to make appointments.

Conclusions

Views on the impact of the Internet were generally positive, but there is a long way to go before its full potential is realised.

\section{IN BRIEF}

- Demonstrates that one half of all dentists have been asked about Internet derived information by patients.

- While accepting that the Internet is a useful source of information, one third of dentists suggested that such information has led to patients seeking more complex or inappropriate care.

- While the great majority of dentists use the Internet to seek information for themselves, three in four never refer patients to the Internet.

- There is an urgent need to help practitioners identify the most useful websites.

\section{COMMENT}

The concept of a patient led NHS has brought into focus the health literacy of patients. There is no doubt that at least for some patients, the Internet will enhance literacy, enabling them to engage fully with their health professionals in decisions relating to their healthcare.

This study is therefore a very timely investigation into the use of the Internet by patients and dentists alike and the impact that its use is having on oral health care in Wales.

Both Wales and England are moving in a parallel direction in terms of reforming NHS dentistry. Key to the reforms is the concept of patients actively participating in decisions regarding their dental care. In this study the authors identified that a considerable proportion of patients were reported to use the Internet as a source of information for this purpose.

In terms of Internet use by dentists, the number of dental practices in Wales that have developed or are considering development of their own websites is encouraging. In the IT enabled dental practice, dentists will in due course be connecting with the rest of the NHS through secure broadband connections. The full use of informatics within dental practices may seem a long way off, however dentistry is within scope. In due course all dental practices will be linked electronically to the rest of the NHS. As the authors identify, some dental practices in Wales are beginning to recognise the potential benefits of information technology in practice administration and online booking. The Internet also opens up new avenues for CPD postgraduate education and research into evidence-based health care.

Further research needs to be undertaken on the impact of social factors on Internet use and the accuracy of the information available. The increased use of the Internet to provide oral health information could potentially lead to a cultural information lag between higher and lower social economic groups and between the elderly and the young. This was clearly identified in the paper by a CDS practitioner whose patients have literacy problems.

This paper has provided a valuable insight into the current use of the Internet in the provision of dental care. It is important that dental informatics research continues as specifications are developed for the procurement of the dental IT systems which will be required for oral health care provision.

A. Jenner, Head of Policy Development, Office of the Chief Dental Officer, Department of Health, London doi: 10.1038/sj.bdj.4813196 